\title{
Pioglitazone does not increase the risk of type II diabetes in patients with bladder cancer: A retrospective study
}

\author{
YOUHONG DONG $^{1}$ and ANPING WANG ${ }^{2}$ \\ Departments of ${ }^{1}$ Oncology and ${ }^{2}$ Neurology, Xiangyang Hospital Affiliated to Hubei University of Medicine, \\ Xiangyang, Hubei 441000, P.R. China
}

Received February 12, 2016; Accepted May 13, 2016

DOI: 10.3892/ol.2016.4566

\begin{abstract}
The aim of the retrospective study was to analyze the effect of pioglitazone on the expression of tumor tissue inflammation factor interleukin (IL)-8, macrophage colony-stimulating factor (M-CSF) and vascular endothelial growth factor (VEGF) of type II diabetes in bladder cancer patients. In addition, whether there was a correlation between pioglitazone and the occurrence of male bladder cancer was also investigated. In total, 42 male cases diagnosed with type II diabetes secondary to bladder cancer were selected. Forty male cases, with simplex type II diabetes but not with bladder cancer, served as the control. Tumor biopsy specimens were collected to detect the expression levels of IL-8, M-CSF and VEGF. The results showed that the expression of IL-8, M-CSF and VEGF of the simplex diabetes group was significantly lower than that of the secondary to tumor group $(\mathrm{P}<0.05)$. The comparison of the two groups in terms of daily dose and time of oral pioglitazone, duration of diabetes, average fasting blood sugar and glycated hemoglobin levels, was not statistically significant. Multivariable logistic regression analysis revealed that the expression levels of IL-8, M-CSF and VEGF were independent risk factors for the occurrence of bladder cancer $(\mathrm{P}<0.05)$, but were not associated with daily dose and time of oral pioglitazone $(\mathrm{P}>0.05)$. In conclusion, oral pioglitazone may not increase the risk of type II diabetes patients with bladder cancer. However, the occurrence of bladder cancer be associated with the increasing expression levels of IL-8, M-CSF and VEGF.
\end{abstract}

\section{Introduction}

The peroxisome proliferator-activated receptor (PPAR) $-\gamma$ agonist can increase insulin sensitivity and first-line regimen

Correspondence to: Dr Anping Wang, Department of Neurology, Xiangyang Hospital Affiliated to Hubei University of Medicine, 15 Jiefang Road, Xiangyang, Hubei 441000, P.R. China E-mail: efqj923@163.com

Key words: pioglitazone, type II diabetes, bladder cancer, interleukin-8, macrophage colony-stimulating factor, vascular endothelial growth factor, retrospective study for type II diabetes (1). However, whether pioglitazone causes male bladder cancer remains to be determined. Previous animal experiments and in vitro cell studies demonstrated the inhibition and promotion of cell proliferation (2). Additionally, clinical retrospective studies have shown that in different countries, with various sample size, there is a correlation between pioglitazone and the occurrence of bladder cancer, albeit there is no significant correlation between the pioglitazone and tumor (3). Consequently, the American FDA added a warning regarding the increasing risk of bladder cancer in the instructions pertaining to the medication (4).

Previous findings have shown that the occurrence of bladder cancer is closely associated with chronic inflammation (5). Bladder cancer tissue can express a high level of interleukin (IL)-8, macrophage colony-stimulating factor (M-CSF) and vascular endothelial growth factor (VEGF), which are correlated significantly with pathological staging and prognosis.

Owing to the high number of diabetic patients in China (6), pioglitazone remains a crucial antidiabetic drug. In the present retrospective study, we analyzed the effect of pioglitazone on the expression of tumor tissue inflammation factor of type II diabetes secondary to bladder cancer, and investigated whether there is a correlation between pioglitazone and the occurrence of male bladder cancer.

\section{Patients and methods}

Patient information. In total, 42 male cases diagnosed as type II diabetes secondary to bladder cancer and hospitalized between October 2012 and October 2015 were consecutively selected. The diagnostic criteria included: i) Prior diabetes disease history, without other malignant tumors; ii) no other malignant tumors before diabetes; and iii) orthotopic bladder cancer. Inclusion criteria for the study were: i) Taking pioglitazone regularly, no complications of serious hypoglycemia and hyperglycemia; ii) no adverse reactions associated with pioglitazone; iii) no urinary system and systemic inflammatory response, such as urinary tract infections, chronic pneumonia; and iv) complete clinical information. Exclusion criteria for the study were: i) Difficult to derive tumor tissue; ii) difficult to control blood sugar, hypoglycemic program was not fixed, serious complications of diabetes such as diabetic nephropathy; and iii) autoimmune diseases. 
The present study was approved by the Ethics Committee of Xiangyang Hospital Affiliation to Hubei University of Medicine (Hubei, China). Informed consent was obtained from the patients or their families. The patients were aged 46-72 years, with an average of $66.3 \pm 13.5$ years. Forty men with simplex type II diabetes but not secondary to bladder cancer, aged 44-73 years, with an average age of $65.8 \pm 12.4$ years, served as the control group. The age comparison between the two groups was not statistically significant $(\mathrm{P}>0.05)$.

Methods. Tumor biopsy specimens were obtained, and enzyme-linked immunosorbent assay (ELISA) was used to detect the expression levels of IL-8, M-CSF and VEGF. Kits were purchased from RD Biological Science and Technology (Minneapolis, MN, USA); microplate was purchased from BioTek (Winooski, VT, USA), model ELX800; the precision pipette and tip were purchased from Gilson, Inc. (Villiers le Bel, France); the constant temperature incubator was purchased from Shanghai Jing Hong Experimental Equipment Co., Ltd. (Shanghai, China), model GNP7200; and a $-80^{\circ} \mathrm{C}$ refrigerator was purchased from Thermo Fisher Scientific (Waltham, MA, USA), model ULT1786-6-V49. The kit used a double antibodystep sandwich ELISA method.

ELISA was performed according to the manufacturer's (RD Biological Science and Technology) instructions. Absorbance (optical density, OD) was measured using a microplate reader at $450 \mathrm{~nm}$.

Observational index. The expression levels of IL-8, M-CSF and VEGF, daily dose and time of oral pioglitazone, duration of diabetes, average fasting blood sugar and glycated
Table I. Comparison of inflammation factor expression levels $(\mu \mathrm{g} / \mathrm{l})$.

\begin{tabular}{|c|c|c|c|}
\hline Group & IL-8 & M-CSF & VEGF \\
\hline $\begin{array}{l}\text { Secondary to } \\
\text { tumor group }\end{array}$ & $0.47 \pm 0.06$ & $0.82 \pm 0.15$ & $29.5 \pm 4.3$ \\
\hline $\begin{array}{l}\text { No secondary to } \\
\text { tumor group }\end{array}$ & $0.26 \pm 0.05$ & $0.33 \pm 0.16$ & $7.46 \pm 2.5$ \\
\hline t-test & 4.625 & 5.532 & 6.634 \\
\hline P-value & 0.039 & 0.024 & 0.013 \\
\hline
\end{tabular}

IL-8, interleukin-8; M-CSF, macrophage colony-stimulating factor; VEGF, vascular endothelial growth factor.

hemoglobin levels, was compared between the two groups. The conventional biochemical methods were used to detect fasting blood sugar and glycated hemoglobin levels, and the average was detected in three consecutive weeks, with almost three-month stable blood sugar.

Statistical analysis. SPSS 20.0 software (SPSS Inc., Chicago, IL, USA) was used for statistical analysis. The data are shown as mean \pm standard deviation, and comparison between groups was made using t-test. Countable data are presented as a percentage, and a comparison was made between groups using the $\chi^{2}$ test. Multivariate regression analysis was performed using the logistic method, screened by the stepback method. $\mathrm{P}<0.05$ was considered to indicate a statistically significant difference.

Table II. Comparison of oral pioglitazone and diabetes control index.

\begin{tabular}{lccccc}
\hline Group & $\begin{array}{c}\text { Daily dose } \\
\text { (mg) }\end{array}$ & $\begin{array}{c}\text { Oral time } \\
\text { (year) }\end{array}$ & $\begin{array}{c}\text { Duration of } \\
\text { diabetes (year) }\end{array}$ & $\begin{array}{c}\text { Mean fasting blood } \\
\text { glucose (mmol/l) }\end{array}$ & $\begin{array}{c}\text { Average glycated } \\
\text { hemoglobin (\%) }\end{array}$ \\
\hline $\begin{array}{l}\text { Secondary to } \\
\text { tumor group }\end{array}$ & $25.6 \pm 4.3$ & $7.2 \pm 2.8$ & $10.5 \pm 3.6$ & $7.6 \pm 2.5$ & $6.8 \pm 1.4$ \\
$\begin{array}{l}\text { No secondary to } \\
\text { tumor group }\end{array}$ & $26.2 \pm 4.5$ & $7.3 \pm 2.6$ & $11.3 \pm 3.8$ & $7.4 \pm 2.6$ & $6.7 \pm 1.3$ \\
t-test & 0.526 & 0.324 & 0.823 & 0.936 & 0.527 \\
P-value & 0.321 & 0.128 & 0.722 & 0.653 & 0.276 \\
\hline
\end{tabular}

Table III. Risk factor analysis of bladder cancer occurrence.

\begin{tabular}{lrrrrr}
\hline Factor & $\beta$ & Wald & P-value & OR & $95 \%$ CI \\
\hline IL-8 & 0.123 & 5.928 & 0.022 & 1.854 & $0.623-2.697$ \\
M-CSF & 0.154 & 6.423 & 0.017 & 1.625 & $0.325-2.532$ \\
VEGF & 0.236 & 5.847 & 0.024 & 1.328 & $0.124-2.863$ \\
Daily dose of pioglitazone & -0.527 & 2.301 & 0.965 & 0.321 & $-0.231-2.527$ \\
Oral pioglitazone time & 0.329 & 2.432 & 0.786 & 0.524 & $-1.523-2.586$ \\
\hline
\end{tabular}

OR, odds ratio; CI, confidence interval; IL-8, interleukin-8; M-CSF, macrophage colony-stimulating factor; VEGF, vascular endothelial growth factor. 


\section{Results}

Comparison of inflammation factor expression levels. The expression levels of IL-8, M-CSF and VEGF in the simplex diabetes group was significantly lower than that in the secondary to tumor group $(\mathrm{P}<0.05)$ (Table I).

Comparison of oral pioglitazone and diabetes control index. Comparison of the two groups in terms of daily dose and time of oral pioglitazone, duration of diabetes, average fasting blood sugar and glycated hemoglobin levels. The differences were of no statistical significance $(\mathrm{P}>0.05)$ (Table II).

Risk factor analysis of bladder cancer occurrence. Logistic regression analysis showed that the expression levels of IL-8, M-CSF and VEGF were independent risk factors of the occurrence of bladder cancer $(\mathrm{P}<0.05)$, but had no association with daily dose and time of oral pioglitazone $(\mathrm{P}>0.05)$ (Table III).

\section{Discussion}

Regarding the correlation between pioglitazone and bladder cancer occurrence, a number of epidemiological clinical retrospective studies (7) have been carried out for different groups, while Japan Takeda Pharmaceuticals, based on the American FDA demands, carried out a 10-year cohort study (7). Midterm analysis revealed that there is no evident correlation between the use of pioglitazone and occurrence of bladder cancer. However, with the increase of time and cumulative doses, bladder cancer risk also increased (medicine exposure time was $>24$ months, when cumulative doses were $>28 \mathrm{~g}$ ). Nevertheless, there is a lack of evidence showing pioglitazone exacerbates the degree of tumor malignancy in bladder cancer patients. A comparison between Asian groups including Taiwan large sample-nested cases and test results showed that long-term use of pioglitazone and rosiglitazone is associated with increased risk of bladder cancer, with bladder cancer risk of patients exposed for 2 years being the highest (8). A Korean retrospective matched case-control study showed that there is no evidence of diabetic patients treated with pioglitazone having increased bladder cancer risk (9). Only the odds ratio (OR) value was comparatively high (2.09), although there was no significant difference compared with those not taking the pioglitazone. In the present study, comparison of the two groups in terms of daily dose and time of oral pioglitazone, duration of diabetes, average fasting blood sugar and glycated hemoglobin levels, showed that the differences were of no statistical significance. Logistic analysis revealed that the expression levels of IL-8, M-CSF and VEGF were independent risk factors for the occurrence of bladder cancer, although there was no association with daily dose and time of oral pioglitazone. The results show that pioglitazone may not increase the incidence of bladder cancer, and the occurrence of bladder cancer may be associated with local tissue inflammation reaction.

IL-8, also known as CXC chemokine, was first identified as a small molecule-secreted protein having the function of chemotaxis and activation on neutrophils (10). It plays an important role in acute and chronic inflammation, chronic obstructive pulmonary disease, asthma, atherosclerosis and other diseases. Recent findings have shown that IL-8 has an intensive angiogenic effect that affects tumor cell proliferation, survival and movement and an important effect on the occurrence, invasion and movement of tumors (11). At present, IL- 8 and its receptor derivatives or inhibitors have become a hot spot in clinical study. Disconnecting the interaction of IL- 8 and its receptor, not only decreases the inflammatory reaction, but also inhibits tumor cell proliferation (12). Macrophage infiltration in tumor tissue, not only has the function of antigen presentation, regulating specific T-cell immunity, secreting effector cell killing targets and killing tumor cells directly, excessively activating macrophages or the tumor initiation factor, but can also promote genomic instability and vascular regeneration (13). M-CSF is highly expressed in bladder cancer tissue, and has a positive correlation with macrophage counts. A number of studies have confirmed that VEGF is capable of promoting endothelial cell proliferation, an essential inducible factor in tumor angiogenesis, and its expression in tissue can reflect angiogenesis activity (14). Normal bladder mucosa epithelial cells have rare VEGF expression, and are only partially expressed in interstitial fibroblasts and endothelial cells. However, bladder carcinoma epithelial, tumor and inflammatory cells have a high expression of VEGF. VEGF through chemotaxis on a variety of immune cells in inflammatory tissue promotes the proliferation of inflammatory tissue endothelial cells, fibroblasts and epithelial cells, leading to tissue cells undergoing a process from mild hyperplasia, atypical hyperplasia, and cell mutation to cancerous hyperplasia (15). In the present study, the expression levels of IL-8, M-CSF and VEGF of the simplex diabetic group was significantly lower than that of the secondary to tumor group.

In conclusion, oral pioglitazone does not increase the risk of type II diabetes in patients with bladder cancer. In addition, the occurrence of bladder cancer may be associated with the increasing expression levels of IL-8, M-CSF and VEGF.

\section{References}

1. Shulman AI and Mangelsdorf DJ: Retinoid x receptor heterodimers in the metabolic syndrome. N Engl J Med 353: 604-615, 2005.

2. Tseng $\mathrm{CH}$ and Tseng FH: Peroxisome proliferator-activated receptor agonists and bladder cancer: lessons from animal studies. J Environ Sci Health C Environ Carcinog Ecotoxicol Rev 30: 368-402, 2012.

3. Kostapanos MS, Elisaf MS and Mikhailidis DP: Pioglitazone and cancer: angel or demon? Curr Pharm Des 19: 4913-4929, 2013.

4. Grygiel-Górniak B: Peroxisome proliferator-activated receptors and their ligands: Nutritional and clinical implications - a review. Nutr J 13: 17, 2014.

5. Zhu YM and Woll PJ: Mitogenic effects of interleukin-8/CXCL8 on cancer cells. Future Oncol 1: 699-704, 2005.

6. Cui R, Qi Z, Zhou L, Li Z, Li Q and Zhang J: Evaluation of serum lipid profile, body mass index, and waistline in Chinese patients with type 2 diabetes mellitus. Clin Interv Aging 11: 445-452, 2016.

7. Lewis JD, Ferrara A, Peng T, Hedderson M, Bilker WB, Quesenberry CP Jr, Vaughn DJ, Nessel L, Selby J and Strom BL: Risk of bladder cancer among diabetic patients treated with pioglitazone: interim report of a longitudinal cohort study. Diabetes Care 34: 916-922, 2011.

8. Hsiao FY, Hsieh PH, Huang WF, Tsai YW and Gau CS: Risk of bladder cancer in diabetic patients treated with rosiglitazone or pioglitazone: a nested case - control study. Drug Saf 36: 643-649, 2013. 
9. Song SO, Kim KJ, Lee BW, Kang ES, Cha BS and Lee HC: The risk of bladder cancer in korean diabetic subjects treated with pioglitazone. Diabetes Metab J 36: 371-378, 2012.

10. Geisinger ML, Michalowicz BS, Hou W, Schoenfeld E, Gelato M, Engebretson SP, Reddy MS and Hyman L: Systemic inflammatory biomarkers and their association with periodontal and diabetes-related factors in the diabetes and periodontal therapy trial (DPTT), a randomized controlled trial. J Periodontol: April 25, 2016 (Epub ahead of print).

11. Liu X, Peng J, Sun W, Yang S, Deng G, Li F, Cheng JW and Gordon JR: G31P, an antagonist against CXC chemokine receptors 1 and 2, inhibits growth of human prostate cancer cells in nude mice. Tohoku J Exp Med 228: 147-156, 2012.

12. Deng F, Wang J, Fan M, Guo Y, Li Y and Shi Q: [Overexpression of IL-8 promotes migration of BT549 breast cancer cells]. Xi Bao Yu Fen Zi Mian Yi Xue Za Zhi 32: 585-589, 2016.
13. Lackner C, Jukic Z, Tsybrovskyy O, Jatzko G, Wette V, Hoefler G, Klimpfinger M, Denk H and Zatloukal K: Prognostic relevance of tumour-associated macrophages and von Willebrand factor-positive microvessels in colorectal cancer. Virchows Arch 445: 160-167, 2004.

14. Infanger M, Kossmehl P, Shakibaei M, Cogoli A, Witzing A, Faramarzi S, Wittfoht W, Paul M and Grimm D: Vascular endothelial growth factor inhibits programmed cell death of endothelial cells induced by clinorotation. J Gravit Physiol 11: 199-200, 2004

15. Chodorowska G, Chodorowski J and Wysokiński A: Vascular endothelial growth factor (VEGF) in physiological and pathological conditions. Ann Univ Mariae Curie Sklodowska Med 59: 8-14, 2004. 